Meta

Journal des traducteurs

Translators' Journal

\title{
Análisis de la función cognitiva de la variación denominativa en la Lexicografía brasileña: patrones conceptuales de variación y distancia semántica entre las variantes
}

\section{Lucimara Alves da Costa et Sabela Fernández Silva}

Volume 63, numéro 2, août 2018

URI : https://id.erudit.org/iderudit/1055148ar

DOI : https://doi.org/10.7202/1055148ar

Aller au sommaire du numéro

\section{Éditeur(s)}

Les Presses de l’Université de Montréal

\section{ISSN}

0026-0452 (imprimé)

1492-1421 (numérique)

\section{Découvrir la revue}

\section{Citer cet article}

da Costa, L. A. \& Fernández Silva, S. (2018). Análisis de la función cognitiva de la variación denominativa en la Lexicografía brasileña: patrones conceptuales de variación y distancia semántica entre las variantes. Meta, 63(2), 467-491. https://doi.org/10.7202/1055148ar
Résumé de l'article

Cet article explore l'utilité cognitive de la variation terminologique, plus particulièrement son rôle dans la construction et transmission des connaissances spécialisées dans les textes. À cette fin, nous analysons la variation dénominative explicite - introduite par des marques discursives d'équivalence - dans un corpus composé de 300 textes du domaine de la lexicographie écrits en portugais brésilien. Nous avons développé une analyse sémantique cognitive en deux étapes. Premièrement, nous avons analysé les modèles de spécification conceptuelle reflétés dans les variantes (Kageura 2002), ce qui nous a permis de visualiser les différents points de vue sur les concepts fournis à travers la variation. Deuxièmement, nous avons analysé la distance sémantique entre le terme de référence et les variantes dénominatives (Fernández-Silva 2016), de façon à quantifier le degré d'informativité de la variation dénominative. Les résultats montrent que la variation reflète la multidimensionnalité de la structuration conceptuelle, où différentes perspectives de catégorisation d'un même concept peuvent cohabiter dans les textes. Par ailleurs, nous avons constaté que dans $85 \%$ des cas analysés, la variation dénominative fournit des informations supplémentaires sur les concepts, concrètement sur les caractéristiques internes et les relations avec d'autres concepts du domaine. En conclusion, cette étude appuie la thèse selon laquelle la variation terminologique aurait une utilité cognitive, car elle permet de mettre en valeur différentes dimensions du contenu conceptuel et, par ce moyen, construire une représentation enrichie des connaissances spécialisées dans les textes. 


\title{
Análisis de la función cognitiva de la variación denominativa en la Lexicografía brasileña: patrones conceptuales de variación y distancia semántica entre las variantes'
}

\author{
LUCIMARA ALVES DA COSTA \\ Pontificia Universidad Católica de Valparaíso, Valparaíso, Chile \\ lucimara.costa@hotmail.com \\ SABELA FERNÁNDEZ SILVA \\ Pontificia Universidad Católica de Valparaíso, Valparaíso, Chile \\ sabela.fernandez@pucv.cl
}

\begin{abstract}
RÉSUMÉ
Cet article explore l'utilité cognitive de la variation terminologique, plus particulièrement son rôle dans la construction et transmission des connaissances spécialisées dans les textes. À cette fin, nous analysons la variation dénominative explicite - introduite par des marques discursives d'équivalence - dans un corpus composé de 300 textes du domaine de la lexicographie écrits en portugais brésilien. Nous avons développé une analyse sémantique cognitive en deux étapes. Premièrement, nous avons analysé les modèles de spécification conceptuelle reflétés dans les variantes (Kageura 2002), ce qui nous a permis de visualiser les différents points de vue sur les concepts fournis à travers la variation. Deuxièmement, nous avons analysé la distance sémantique entre le terme de référence et les variantes dénominatives (Fernández-Silva 2016), de façon à quantifier le degré d'informativité de la variation dénominative. Les résultats montrent que la variation reflète la multidimensionnalité de la structuration conceptuelle, où différentes perspectives de catégorisation d'un même concept peuvent cohabiter dans les textes. Par ailleurs, nous avons constaté que dans $85 \%$ des cas analysés, la variation dénominative fournit des informations supplémentaires sur les concepts, concrètement sur les caractéristiques internes et les relations avec d'autres concepts du domaine. En conclusion, cette étude appuie la thèse selon laquelle la variation terminologique aurait une utilité cognitive, car elle permet de mettre en valeur différentes dimensions du contenu conceptuel et, par ce moyen, construire une représentation enrichie des connaissances spécialisées dans les textes.
\end{abstract}

\begin{abstract}
This article explores the cognitive function of terminological variation, specifically its contribution to the construction and transfer of specialized knowledge in texts. For that purpose, explicit term variation - introduced by equivalence markers - was analysed in a corpus of 300 texts about lexicography written in Brasilian Portuguese. A cognitive semantic analysis was carried out in two steps: first, a description of the conceptual specification patterns (Kageura 2002) of variants allowed us to identify how different viewpoints on the concepts are provided by terminological variation. Second, variants were classified according to the degree of semantic distance from the base term (Fernández-Silva 2016) in order to quantify the informativity degree of term variation. Results showed that term variation reflects the multidimensionality of conceptual structuring, where different perspectives on the same concept cohabitate in texts. Furthermore, $85 \%$ of term variants provide additional information on the concept, specifically about
\end{abstract}


its internal features and the relationships that it establishes with other concepts within the field. In conclusion, this study supports the thesis that term variation can be used as a cognitive device. It makes it possible to depict different aspects of the conceptual content and, in turn, contributes to a richer representation of knowledge in texts.

\section{RESUMEN}

En este artículo exploramos la función cognitiva de la variación terminológica y, específicamente, su papel en la construcción y transmisión del conocimiento especializado en los textos. Para ello, analizamos la variación denominativa explícita - identificada mediante marcadores discursivos de equivalencia - en un corpus de 300 textos sobre lexicografía escritos en portugués de Brasil. Llevamos a cabo un análisis semánticocognitivo en dos etapas: primero, analizamos los patrones de especificación conceptual de las variantes (Kageura 2002), lo cual nos permitió describir los distintos puntos de vista sobre los conceptos proporcionados a través de la variación. En segundo lugar, analizamos la distancia semántica entre el término de referencia y las variantes denominativas (Fernández-Silva 2016), lo cual nos permitió cuantificar el grado de informatividad de la variación denominativa. Los resultados mostraron, por un lado, que la variación denominativa refleja el carácter multidimensional de la estructuración conceptual, donde conviven diferentes perspectivas de categorización de un mismo concepto; y por otro, que las variantes denominativas aportan información adicional sobre los conceptos en un $85 \%$ de los casos analizados, concretamente información sobre las características internas del concepto y sobre su relación con otros conceptos del ámbito. Este estudio apoya la tesis de que la variación denominativa desempeña una función cognitiva, puesto que permite poner de relieve diferentes aspectos del contenido conceptual y, de este modo, construir una representación enriquecida del conocimiento en los textos.

\section{MOTS-CLÉS/ KEYWORDS/ PALABRAS-CLAVE}

variation terminologique, lexicographie brésilienne, variation dénominative explicite, utilité cognitive

term variation, lexicography in Brasil, explicit denominative variation, cognitive function variación terminológica, lexicografía brasileña, variación denominativa explícita, función cognitiva

\section{Introducción}

La variación denominativa, o la presencia de varias denominaciones para referirse a un mismo concepto, es un fenómeno plenamente aceptado por las teorías modernas de la terminología. Desde una óptica descriptiva, que busca comprender el rol de la terminología en la representación y comunicación del conocimiento especializado en una diversidad de contextos, teorías como la Socioterminología (Gaudin 1993), la Teoría Comunicativa de la Terminología (Cabré 1999), la Teoría Sociocognitiva (Temmerman 2000) o la Terminología basada en marcos (Faber, Martínez, et al. 2006) reconocen que la variación es inherente a la unidad terminológica y tiene un comportamiento y función específicos que obedecen a los parámetros propios de la comunicación especializada (Freixa 2006).

Muchos estudios derivados de estos nuevos enfoques han investigado la variación en relación con distintos factores dialectales y funcionales, como el registro (Faulstich 1998, 2000), el nivel de especialización (Freixa 2002; Ciapuscio 2003; Jesus y Barros 2005; Krieger y Santiago 2014), el estatus socioprofesional (Bertaccini y Matteuci 2005), el área de especialidad (Nadin y Silva 2008) o el canal (Seghezzi 2011). 
Otros autores han estudiado la variación desde una perspectiva cognitiva (Rogers 2004; Bowker y Hawkins 2006; Fernández-Silva, Freixa, et al. 2011; Tercedor 2011; Pecman 2014; Fernández-Silva 2016). Desde esta perspectiva, la variación es reflejo de los procesos dinámicos de categorización y estructuración del conocimiento especializado y, por tanto, tiene una motivación cognitiva (Tercedor 2011: 182). Por otro lado, la utilización de diferentes variantes para un mismo concepto permite resaltar diferentes aspectos de su contenido y contribuye "al proceso continuo de construcción del conocimiento” (Pecman 2014: 10). Así, la variación desempeñaría una función cognitiva tanto en la producción como en la recepción, desde el momento en que se construye el conocimiento especializado en la mente del autor hasta que se accede a él por medio de las distintas variantes empleadas en el texto.

La variación denominativa intratextual, generada por un mismo autor en un mismo texto, se ha vinculado mayormente a factores estilísticos o textuales (Fernández Polo 1999; Freixa 2005). El uso de distintas variantes facilita la lectura y contribuye a la producción de textos "estilísticamente aceptables" (Freixa 2005: 10). Pero más allá de la función estilística, que es la más consciente para los propios especialistas (Freixa 2005), se ha comenzado a explorar la función cognitiva y retórica de este tipo de variación y su papel en la construcción del conocimiento especializado en los textos (Pecman 2012, 2014; Fernández-Silva 2016).

Con objeto de profundizar en este aspecto, en este trabajo nos hemos planteado analizar la función cognitiva de la variación denominativa explícita en el área de la Lexicografía. A partir de un corpus de textos académicos sobre Lexicografía publicados en portugués de Brasil (Costa 2015), se analizaron las variantes denominativas introducidas en los textos mediante marcadores discursivos de equivalencia (Suárez 2004). A continuación, se procedió a analizar la información conceptual expresada por las variantes y la distancia semántica con respecto al término de referencia (Fernández-Silva 2016) con el objetivo de describir los patrones conceptuales de variación denominativa y la función cognitiva de este recurso.

El artículo está estructurado en las siguientes partes: tras esta introducción, presentamos el marco teórico que sustenta nuestra investigación, donde abordamos la variación denominativa desde una perspectiva cognitiva, y revisamos distintas propuestas de clasificación semántica para analizar la función cognitiva de la variación. En el apartado 3 presentamos la metodología, seguida de los resultados (apartado 4) y finalizamos con las conclusiones, limitaciones y trabajo futuro.

\section{Marco teórico}

A continuación presentamos los fundamentos teóricos para el estudio de la variación terminológica desde una perspectiva cognitiva y revisamos algunas propuestas de clasificación semántica de la variación en las que nos basamos para elaborar nuestra propuesta de análisis de la función cognitiva de la variación.

\subsection{La variación terminológica desde una perspectiva cognitiva}

Hoy en día el estudio de la variación en terminología cuenta con una sólida fundamentación teórica y con abundantes estudios descriptivos que han abordado este fenómeno desde el plano lingüístico, sociocomunicativo y cognitivo de la unidad 
terminológica. Desde el plano cognitivo, la variación terminológica se ha vinculado con los procesos dinámicos de categorización y estructuración del conocimiento especializado y el carácter situado de la cognición. Las propuestas teóricas de corte cognitivo, como la Teoría Sociocognitiva de la Terminología (Temmerman 2000), la Teoría Comunicativa de la Terminología (Cabré 1999, 2008; Fernández-Silva, Freixa, et al. 2011) o la Teoría basada en Marcos (Faber, Martínez, et al. 2006; Faber 2012) han integrado modelos semánticos procedentes de las ciencias cognitivas en sus explicaciones sobre la formación y estructuración de los conceptos especializados, las características de los sistemas conceptuales y su denominación por medios lingüísticos. Así, la teoría del prototipo y nivel básico (Rosch 1978; Temmerman 2000), la teoría de la metáfora conceptual (Lakoff y Johnson 1980; Temmerman 2000; Tercedor y López 2012), la semántica de marcos (Fillmore 1985; Faber, Martínez, et al. 2006) o la teoría de la cognición situada (Barsalou 2003; Tercedor 2011) rebaten la visión objetivista del conocimiento, defendida por la terminología tradicional, donde los conceptos son universales y unívocos, las estructuras conceptuales son estáticas y el lenguaje no desempeña ningún papel en la formación de los conceptos. Los principios que comparten las propuestas terminológicas de corte cognitivista se pueden resumir en los siguientes aspectos:

1) El conocimiento especializado no es un reflejo objetivo del mundo sino el resultado de nuestra comprensión del mundo; está mediado por la experiencia de nuestros cuerpos, nuestra experiencia física con el entorno y nuestra interacción como seres sociales.

2) El pensamiento no se reduce a operaciones mentales lógicas de manipulación de elementos abstractos, sino que opera en relación con otras capacidades cognitivas como la percepción, la atención, la memoria, la actividad sensoriomotriz, la emoción o la interacción social.

3) El conocimiento especializado se genera en el interior de una comunidad circunscrita a un contexto temporal, socioprofesional y cultural determinado, por lo que los conceptos y estructuras conceptuales pueden sufrir modificaciones de acuerdo a estos factores contextuales.

La adscripción a estos postulados implica reconocer que la variabilidad está presente en todas las etapas del proceso: la percepción de la realidad, la formación y estructuración de conceptos y su verbalización en las distintas situaciones de comunicación especializada. En este escenario, la variación terminológica no solo tiene una motivación cognitiva sino que también es funcional en el proceso de construcción del conocimiento especializado en los textos.

Partiendo de este supuesto, Temmerman (2000) analiza cómo algunas categorías de las ciencias naturales presentan una estructura prototípica para cuya definición no basta con indicar la posición en una estructura lógico-ontológica y las características distintivas. Por el contrario, se deben tener en cuenta otros elementos estructurales tales como el origen de la comprensión; las dimensiones constituyentes de la estructura intracategorial; la perspectiva o posición desde la que se observa la categoría en relación con otras dentro de la estructura intercategorial y la intención del emisor del mensaje, que "determinará qué elementos informativos se explicitarán en la descripción de la categoría” (Temmerman 2000: 74). Del mismo modo, la polisemia y la sinonimia también se asocian con la estructura prototípica de algunos conceptos, que manifiestan una tendencia natural a acumular varios sentidos. Desde el punto 
de vista diacrónico, la polisemia es el resultado de una innovación tecnológica o sociológica de la realidad, de un cambio en la comprensión del concepto o de sus medios de expresión. La cuasisinonimia también es el resultado natural de la evolución del conocimiento y de una búsqueda por la "precisión en la expresión" (Temmerman 2000: 132). La existencia de varios cuasisinónimos refleja distintas perspectivas en la comprensión de un mismo concepto y están a disposición del usuario para que elija aquella que encaja mejor con su comprensión en una determinada situación.

La Teoría Comunicativa de la Terminología constituye una propuesta teórica que "contempla la variación lingüística en toda su dimensionalidad" y "asume un modelo variacionista y textualista que permite su descripción a todos los niveles lingüísticos y discursivos" (Cabré 1999: 106). Diversos estudios enmarcados en esta teoría han explorado la dimensión cognitiva de variación y su relación con otros factores comunicativos y lingüísticos. Freixa (2002), en su estudio pionero sobre variación terminológica, estableció una tipología de causas de variación en las que se distinguen las causas dialectales, funcionales, discursivas, interlingüísticas y cognitivas. Estas últimas están relacionadas con la imprecisión conceptual, la voluntad de distanciación ideológica entre autores o a diferencias en la percepción o comprensión de la realidad o en el "abordaje de un mismo concepto desde puntos de vista diferentes" (Freixa 2002: 338). Más adelante, Fernández-Silva (2011) desarrolla una tipología de factores cognitivos de variación, entre las que se distinguen factores sistémicos -relacionados con la flexibilidad de los conceptos y la multidimensionalidad de los sistemas conceptuales- y factores contextuales, relacionados con diferencias de conceptualización entre grupos de usuarios, en un mismo usuario en distintas situaciones o en el interior de un mismo texto.

Desde esta perspectiva, la variación denominativa no solo tiene una motivación cognitiva sino que también desempeña una función cognitiva, puesto que la convivencia de diferentes denominaciones para un mismo concepto permite poner de relieve diferentes aspectos de su contenido y, de este modo, construir una representación enriquecida del concepto. De este modo, la variación intratextual no solo tiene una función estilística orientada a evitar la repetición, sino que también refleja el modo en que el emisor construye el conocimiento por medios lingüísticos y sirve como mecanismo cognitivo para explicar los conceptos a lo largo del texto (Pecman 2014; Fernández-Silva 2016).

Finalmente, la Terminología basada en Marcos (Faber, Márquez, et al. 2005; Faber, Martínez, et al. 2006) presenta una propuesta de representación conceptual basada en la semántica de marcos (Fillmore 1985) destinada a la creación de bases de datos terminológicos multilingües. De acuerdo con las autoras, los modelos jerárquicos tradicionales no reflejan la organización real de los conceptos ni el dinamismo de la categorización. Los productos terminológicos deben ser elaborados de tal modo que permitan representar el carácter multidimensional, flexible y situado de la estructuración conceptual. Esto incluye la variación conceptual y denominativa, ya que este recurso refleja las diversas posibilidades de aproximarse a una misma realidad y es una herramienta útil para acercar el conocimiento a diferentes audiencias con necesidades comunicativas particulares (Tercedor y López 2012). 


\subsection{Propuestas de clasificación semántica para medir la función cognitiva de la variación denominativa}

En este apartado revisaremos dos propuestas de clasificación semántica de la variación que pueden servir como antecedente para analizar la función cognitiva de este recurso. Estas propuestas comparten una visión flexible sobre la categorización y estructuración conceptuales, lo cual implica reconocer que la equivalencia semántica total entre variantes -conocida en lingüística general como sinonimia absoluta- es solo una de las posibles situaciones de variación. En efecto, como señala Freixa (2002: 351), "los términos no solo denominan sino que también significan", de modo que unidades terminológicas con significados distintos pueden ser empleadas como variantes de un mismo concepto.

La relatividad sinonímica entre variantes aparece ampliamente recogida en la bibliografía bajo distintos nombres: semisinónimos (Kocourek 1991; Contente y Magalhães 2005), sinónimos parciales o relativos (Suárez 2004), sinónimos parciales o cuasi-equivalentes (Diki-Kidiri 2008), cuasisinónimos (Arnz y Picht 1995; Temmerman 2000), variantes con consecuencias cognitivas (Fernández-Silva 2011), etc. También encontramos clasificaciones semánticas de la variación (Wüster 1979/1998) que adoptan como criterio el grado de equivalencia semántica de los distintos componentes de significado (por ejemplo, significado denotativo y connotativo) y la intercambiabilidad contextual. Sin embargo, la clasificación semántica de Freixa (2002) constituye la primera propuesta exhaustiva que asocia el tipo de cambio formal con el grado de equivalencia conceptual entre las variantes y distingue claramente la variación en dos planos de contenido del término: el plano semántico y el plano conceptual. Esta clasificación parte de la premisa de que las diferentes variantes de un concepto reflejan "una selección concreta de rasgos del conjunto completo de rasgos semánticos de diferentes niveles que constituye un concepto” (Freixa 2002: 295). En la Figura 1 se muestra la propuesta de clasificación, que presenta las siguientes características (Freixa 2002: 295-297):

\section{FIGURA 1}

Propuesta de clasificación semántica de la variación denominativa (Freixa 2002)

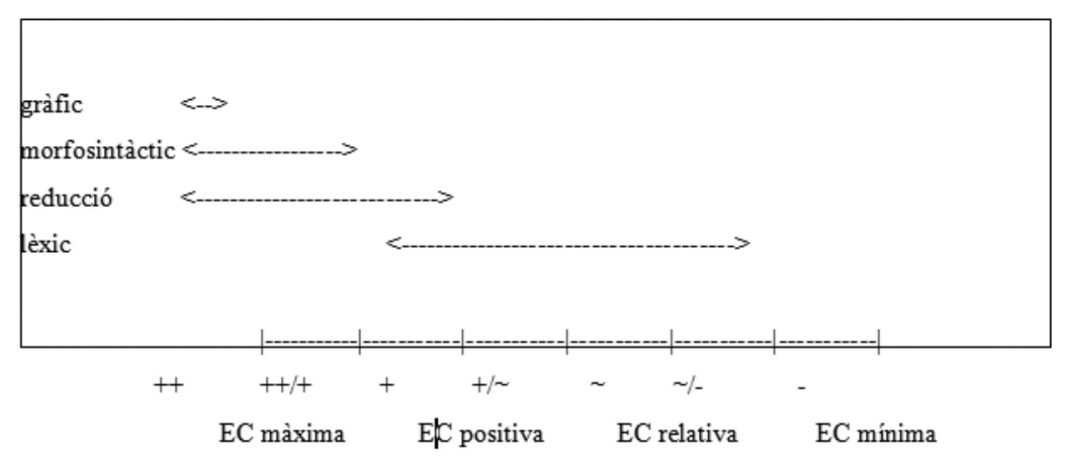

1) La relación de equivalencia conceptual (EC) entre dos denominaciones se entiende como un continuo que va de la EC máxima a la EC mínima, dentro del cual se distinguen cuatro grados (EC máxima, positiva, relativa y mínima) y zonas fronterizas entre ellas. 
2) No todos los cambios denominativos tienen la misma incidencia en el contenido conceptual, por lo que el tipo de cambio formal es un criterio válido para abordar la clasificación semántica y distinguir entre segmentos del eje de equivalencia conceptual.

Así, como se observa en la figura, los cambios gráficos (por ejemplo, alternancia entre término y símbolo o sigla) presentan un grado de equivalencia conceptual máxima, mientras que los cambios léxicos (ya sea en la base, extensión o ambos) podría ir desde equivalencia positiva a mínima, según si las denominaciones difieren por la presencia, ausencia o cambio de un rasgo conceptual no distintivo (EC máxima); de un rasgo conceptual distintivo (EC máxima/relativa); según si la alternancia del rasgo distintivo comporta variación conceptual (EC relativa); o si las denominaciones remiten a conceptos distintos y pertinentes dentro del área de especialidad (EC mínima).

Si bien esta clasificación presenta algunos problemas al correlacionar el tipo de cambio formal con el grado de equivalencia conceptual, creemos que recoge algunos elementos importantes para analizar la función cognitiva de la variación, ya que permite analizar qué aspectos del contenido conceptual aparecen reflejados en las denominaciones y propone un eje continuo de equivalencia que permitiría cuantificar la función cognitiva de la variación, partiendo de la premisa de que cuanto mayor sea el grado de equivalencia conceptual, menor será la función cognitiva. Estos dos elementos se retomarán en nuestra propuesta de análisis de la función cognitiva basada en la distancia semántica.

Por otro lado, Suárez (2004) realiza otra propuesta de clasificación de la variación denominativa (VD) explícita basada en las relaciones semánticas entre variantes y en el orden de aparición. Si bien la autora no se refiere explícitamente a la función cognitiva de la variación, sí admite que esta variación "tiene consecuencias cognitivas importantes con respecto a la planificación del emisor y a las consecuencias interpretativas del receptor" (Suárez 2004: 178). La autora propone analizar la relación semántica desde dos perspectivas opuestas, equivalencia y diferencia, determinando así los componentes comunes y diferenciales del contenido de las variantes. Como señalaba anteriormente, este análisis tiene en cuenta qué rasgos del concepto aparecen mencionados en cada variante y, lo que es más relevante desde el punto de vista de la progresión del conocimiento en el texto, el orden en el que esta información aparece desplegada. Como resultado, la autora propone tres tipos de variación denominativa explícita:

1) Por expansión, cuando una unidad léxica condensada se reformula mediante una unidad léxica más desarrollada y transparente, que añade rasgos que no estaban presentes en la unidad léxica condensada;

2) por reducción, cuando la unidad más desarrollada aparece en primer lugar, seguida de una unidad léxica más condensada, experimentando así una condensación en sus rasgos o componentes semánticos;

3) por refocalización, cuando la variante denominativa en segundo lugar activa rasgos diferentes con respecto a la primera unidad, mostrando así un punto de vista diferente sobre el objeto denominado.

En síntesis, los avances teóricos y descriptivos en torno al estudio de la variación terminológica nos permiten plantear la hipótesis de la función cognitiva de la variación terminológica y su papel en la construcción y transmisión del conocimiento 
especializado en los textos. Por ello, el presente estudio se plantea como objetivo describir la cantidad y tipo de información sobre los conceptos especializados reflejados a través de la variación denominativa explícita en un corpus de textos sobre lexicografía brasileña.

\section{Metodología}

En este apartado describimos los procedimientos metodológicos llevados a cabo para: a) la selección de los términos y detección de las variantes denominativas en corpus; y b) el análisis semántico-cognitivo de la variación de acuerdo a la información conceptual y a la distancia semántica entre variantes.

\subsection{Selección de los términos y detección de las variantes denominativas}

El análisis de la variación denominativa se realizó a partir de un corpus compuesto por 300 textos especializados sobre Lexicografía (45 millones de palabras), pertenecientes a distintos géneros académicos (artículos, reseñas, monografías, tesis, etc.), escritos por autores brasileños o extranjeros en portugués de Brasil en el periodo 1980-2013 (Costa 2015). ${ }^{2}$

Para la extracción de los términos del corpus combinamos los criterios de frecuencia y de estructura morfosintáctica. Con los programas Wordsmith Tools y Terminus ${ }^{3}$ obtuvimos listados de n-gramas organizados por frecuencia, de los que filtramos aquellos con una media de 0,01 de frecuencia relativa (288 ocurrencias) en el corpus. En cuanto a los patrones morfosintácticos, consideramos unidades monoléxicas y poliléxicas de hasta 3 constituyentes (Figura 2) con las siguientes estructuras: $\mathrm{N}$ (ej. macroestrutura); $\mathrm{V}$ (lematizar); $\mathrm{N}+\mathrm{A}+(\mathrm{A})$ (lexicografía infantil, dicionário escolar infantil); $\mathrm{N}+(\mathrm{A})+\mathrm{SP}$ (marca de uso; dicionário geral de língua). Según estos criterios, cruzamos las listas obtenidas con ambos programas y obtuvimos una lista provisional de 500 términos de Lexicografía.

FIGURA 2

Extracción de candidatos a términos con el programa Terminus

\begin{tabular}{|c|c|c|c|}
\hline \multicolumn{4}{|c|}{$\begin{array}{l}\text { Análisis de corpus } \\
\text { Paso } 2 \text { de } 2 \\
\text { Extracción de n-gramas } \\
\text { Results: } 501 \\
\begin{array}{|l|}\text { Validar candidatos seleccionados } \\
\end{array}\end{array}$} \\
\hline No & Unidades & Frecuencia absoluta & Frecuencia relativa \\
\hline 1) $\square$ & da língua portuguesa & 968 & 0.00028282 \\
\hline 2) 0 & rio de janeiro & 582 & 0.00017004 \\
\hline 3) $\square$ & pares de línguas & 454 & 0.00013264 \\
\hline 4) $\square$ & recebeu para os & 424 & 0.00012388 \\
\hline 5) 0 & os respectivos pares & 424 & 0.00012388 \\
\hline 6) 0 & línguas a seguinte & 424 & 0.00012388 \\
\hline 7) $\square$ & ponto de vista & 372 & 0.00010869 \\
\hline 8) 0 & apresenta a ei & 315 & 0.00009203 \\
\hline 9) $\square$ & dicionário da língua & 291 & 0.00008502 \\
\hline 10) $\square$ & português do brasil & 274 & 0.00008005 \\
\hline 11) $\bigcirc$ & não faz nenhuma & 227 & 0.00006632 \\
\hline 12) 0 & sala de aula & 227 & 0.00006632 \\
\hline 13) $\square$ & nenhuma referência à & 225 & 0.00006574 \\
\hline 14) $\square$ & referência à ei & 225 & 0.00006574 \\
\hline 15) $\bigcirc$ & faz nenhuma referência & 225 & 0.00006574 \\
\hline 16)凹 & dicionário de língua & 203 & 0.00005931 \\
\hline 17) $\square$ & uso de dicionários & 198 & 0.00005785 \\
\hline 18) 0 & uso do dicionário & 192 & 0.00005610 \\
\hline 19) $₫$ & dicionários de língua & 185 & 0.00005405 \\
\hline
\end{tabular}


Para la detección de la variación denominativa explícita, aquella que "se da a través de marcadores discursivos que vehiculan equivalencia” (Suárez 2004: 2), procedimos a realizar búsquedas a partir de los marcadores con la herramienta de extracción de concordancias de Wordsmith Tools. Para ello, se partió inicialmente de la lista de marcadores de variación denominativa incluida en Suárez (2004), que se fue ampliando mediante el análisis de los contextos de aparición de los términos y sus variantes. En la Tabla 1 se muestra la lista final de marcadores de variación denominativa incluidos en nuestro análisis y su frecuencia en el corpus.

TABLA 1

Marcadores discursivos de reformulación

\begin{tabular}{|c|c|c|c|}
\hline Marcadores & Frec. (\%) & Marcadores & Frec. $(\%)$ \\
\hline ou & $32(23 \%)$ & referido como/ que se refere & $5(4 \%)$ \\
\hline chamado (de) & $27(19 \%)$ & chamada também de & $4(3 \%)$ \\
\hline()$/[]$ & $16(12 \%)$ & entendido como & $3(2 \%)$ \\
\hline é (também) denominado & $15(11 \%)$ & emprega-se também & $2(1 \%)$ \\
\hline sinônimo de & $7(6 \%)$ & pode ser considerado & $2(1 \%)$ \\
\hline também conhecido como & $6(4 \%)$ & como & $2(1 \%)$ \\
\hline$=$ & $6(4 \%)$ & denominado como & $2(1 \%)$ \\
\hline o mesmo que & $6(4 \%)$ & oscila entre & $2(1 \%)$ \\
\hline que é & $3(2 \%)$ & darei preferência (a) & $2(1 \%)$ \\
\hline
\end{tabular}

La búsqueda mediante marcadores nos permitió detectar la(s) variante(s) denominativa(s) asociadas a un determinado término de referencia. Consideramos término de referencia aquel que aparecía en el primer término de la enumeración y variantes denominativas a todas las sucesivas denominaciones introducidas por marcadores, a menos que el autor manifestara explícitamente la preferencia por otra denominación lo cual sucedió en solo dos casos, como se muestra en el ejemplo 1. Encontramos grupos denominativos - conjunto de denominaciones para un mismo concepto - de 2 hasta 6 elementos, como se muestra en el ejemplo 1:

1) Como se observa, a terminologia não é fixa nas línguas estrangeiras e em português oscila entre ${ }^{(\mathrm{VDI})}$ dicionário escolar, ${ }^{(\mathrm{VD} 2)}$ infantil, ${ }^{(\mathrm{VD} 3)}$ escolar infantil, (VD4) de iniciação, ${ }^{(\mathrm{VD} 5)}$ pré-dicionários. Como já apontado anteriormente, darei preferência à utilização do termo ${ }^{(\mathrm{TR})}$ dicionário infantil.

(Zavaglia 2010: 40-41)

En algunos casos, los marcadores nos permitieron identificar variantes interlingüísticas, tal y como se muestra en el ejemplo 2. Sin embargo, estas fueron descartadas ya que en este trabajo solo nos centramos en el análisis de las variantes intralingüísticas (en el ejemplo, rótulos y marcas de uso). De este modo, se obtuvo un listado final de 100 variantes denominativas, que serán las que analizaremos en este trabajo.

2) Geralmente, os ${ }^{(\mathrm{TR})}$ rótulos (termo empregado por Borba 2003) são denominados ${ }^{(\mathrm{VD1})}$ marcas de uso no português (cf. Strehler 1998), (VD2) marcas no espanhol, ${ }^{(\mathrm{VD} 3)}$ marques ou ${ }^{(\mathrm{VD} 4)}$ marques d'usage no francês, ${ }^{(\mathrm{VD} 5)}$ labels no inglês.

(Welker 2004: 130) 


\subsection{Análisis semántico-cognitivo de la variación}

Para analizar la función cognitiva de la variación denominativa explícita llevamos a cabo un análisis semántico-cognitivo encaminado a analizar la información conceptual aportada por la variación y la distancia semántica entre las variantes. La hipótesis de trabajo detrás de este análisis es que mayor será la función cognitiva de la variación cuanta mayor y más variada información sobre los conceptos se proporcione mediante la coexistencia de las variantes.

Dividimos este análisis en dos fases: en primer lugar, analizamos los patrones conceptuales de variación denominativa, basándonos en las relaciones intratérmino y los patrones de especificación conceptual de Kageura (2002); en segundo lugar, analizamos la distancia semántica entre el término de referencia y las variantes denominativas, para lo cual utilizamos la clasificación de Fernández-Silva (2016). A continuación, describimos con más detalle ambos métodos de análisis.

\subsubsection{Patrones conceptuales de variación denominativa}

En este análisis, las variantes se clasificaron según el patrón de especificación conceptual reflejado en la denominación, es decir aquella dimensión del contenido conceptual que se considera más relevante y permite diferenciarlo de otros conceptos cohipónimos. Para ello, los términos se interpretan como combinaciones más características de los conceptos dentro del sistema conceptual. Cada término refleja información sobre el concepto genérico al que pertenece y sobre la(s) característica(s) distintiva(s). Si se analiza la relación existente entre estas informaciones (relación intratérmino), se obtiene el patrón de especificación conceptual de cada variante. Así, en los términos poliléxicos (dicionário de aprendizagem), el núcleo representa el concepto genérico (un tipo de obra lexicográfica), el modificador la característica que define al concepto en cuestión (la actividad de aprender), y la relación entre núcleo y modificador (relación intratérmino) nos permite identificar que en esta variante se selecciona la función del diccionario como la especificación conceptual que distingue este diccionario de otro tipo de obras. En el caso de los términos monoléxicos, se analiza la relación intratérmino entre los diferentes formantes (por ejemplo, en macroestructura el concepto genérico vendría representado en el lexema estrutura y la característica distintiva a través del prefijo macro-, que indicaría la especificación conceptual de tamaño). En el caso de las unidades con más de dos constituyentes, se describieron todas las relaciones intratérmino pero se le asignó únicamente la especificación conceptual de la relación intratérmino principal. Por ejemplo, en la variante dicionário escolar mini, se describieron dos relaciones intratérmino destino y tamaño, pero se le asignó el patrón de especificación conceptual de tamaño).

Como decíamos anteriormente, empleamos la clasificación de Kageura (2002) que distingue 25 relaciones intratérmino y 20 patrones de especificación conceptual que se agrupan en 6 puntos de vista. Esta clasificación, que en la obra de Kageura (2002) se empleó para analizar los patrones de formación de términos de ciencias de la información, sufrió algunas modificaciones al aplicarlas a nuestros datos, que consistieron básicamente en la eliminación de algunos patrones de especificación genéricos (por ej. especificación de los elementos complementarios, que agrupa las relaciones objeto afectado, producto, medio y manera fue sustituida por especificación del objeto afectado, producto y manera respectivamente); la inclusión de patrones no 
contemplados (concretamente, la especificación del tamaño, en el punto de vista de los atributos internos); o la eliminación de algunas categorías que no aparecieron representadas en nuestros datos (por ejemplo, la especificación de manera estática o medio). En la tabla 2 se muestra la clasificación empleada en nuestro estudio.

TABLA 2

Relaciones intratérmino y patrones de especificación conceptual (adaptado de Kageura 2002)

\begin{tabular}{|c|c|c|}
\hline Punto de vista & Patrón de especificación & Relación(es) intratérmino \\
\hline PDV Igualdad & Esp. de yuxtaposición & Yuxtaposición \\
\hline \multirow[t]{8}{*}{ PDV Funcional } & Esp. del rol & Rol \\
\hline & Esp. del objeto afectado & Objecto afectado \\
\hline & Esp. de la manera & Manera \\
\hline & \multirow[t]{2}{*}{ Esp. de la función } & Función \\
\hline & & $\begin{array}{l}\text { Función+ contenido informativo y } \\
\text { representación+ origen }\end{array}$ \\
\hline & Esp. del uso & Uso \\
\hline & \multirow[t]{2}{*}{ Esp. del destino } & Destino \\
\hline & & Destino+tamaño \\
\hline \multirow[t]{5}{*}{ PDV Parte/todo } & \multirow[t]{2}{*}{ Esp. del todo } & Partitiva \\
\hline & & Partitiva+lugar \\
\hline & Esp. de los constituyentes & Constituyentes \\
\hline & \multirow{2}{*}{$\begin{array}{l}\text { Esp. del contenido informativo y } \\
\text { representación }\end{array}$} & Contenido informativo y representacíon \\
\hline & & Cantidad+ conten. inf. y repr.+ alcance \\
\hline \multirow{5}{*}{$\begin{array}{l}\text { PDV de los } \\
\text { atributos } \\
\text { internos }\end{array}$} & Esp. de atributos formales & Atributos formales \\
\hline & Esp.de naturaleza & Naturaleza \\
\hline & Esp. de concepto atribuido & Concepto atribuido \\
\hline & Esp. de la cantidad & Cantidad \\
\hline & Esp. de tamaño & Tamaño \\
\hline \multirow{5}{*}{$\begin{array}{l}\text { PDV relacional/ } \\
\text { externo }\end{array}$} & Esp. de estatus & Estatus \\
\hline & Esp. de lugar & Lugar \\
\hline & Esp. de tiempo & Tiempo \\
\hline & Esp. de origen & Origen \\
\hline & Esp. de alcance & Alcance \\
\hline
\end{tabular}

Para llevar a cabo la descripción de los patrones de variación, agrupamos las variantes en clases conceptuales. Así, cada grupo denominativo (término de referencia y variantes denominativas explícitas), se trató como un concepto que, a su vez se clasificó según la clase conceptual a la que pertenece. Este agrupamiento nos permitió describir el comportamiento denominativo de todos los conceptos pertenecientes a una clase conceptual y extraer tendencias en el comportamiento de la variación.

\subsubsection{Distancia semántica entre variantes}

El segundo paso de nuestro análisis consistió en comparar la distancia semántica entre el término de referencia y las variantes denominativas a fin de cuantificar el grado de informatividad de la variación denominativa. La hipótesis detrás de este supuesto es que, cuanta mayor es la distancia semántica entre el término de referencia y las variantes denominativas, mayor es la funcionalidad cognitiva de este recurso. 
Para ello, empleamos la clasificación de Fernández-Silva (2016), donde se identifican 9 tipos de variación denominativa intratextual agrupados en tres distancias: máxima, media y mínima (Tabla 3).

La mínima distancia se daría entre variantes que no difieren en el contenido conceptual representado, ya sean sinónimos léxicos u otros tipos de variantes formales (item lexical/item léxico). La distancia media se daría entre términos que difieren en la cantidad y tipo de características distintivas representadas. Dentro de este grupo se incluirían todas las variantes que conllevan un cambio en el modificador, como las reducciones -donde se suprime la característica distintiva como recurso anafórico, como macroestrutura/macro-; la variación donde se produce un cambio, inclusión o supresión de una característica no definitoria (dicionário infantil/ dicionário escolar infantil) y las variantes donde se modifica una característica definitoria (dicionário de língua/dicionário semasiológico). En estos tres casos se produce un cambio en la dimensión o rasgo del concepto que se visibiliza en la denominación (variación intracategorial).

TABLA 3

Propuesta de clasificación basada en la distancia semántica (Fernández-Silva 2016)

\begin{tabular}{|l|l|l|}
\hline \multirow{4}{*}{ Distancia máxima } & Cambio de configuración conceptual & Verbete/artigo lexicográfico \\
\cline { 2 - 3 } & Hiperonimia/hiponimia & Rótulos/ marcas de uso \\
\cline { 2 - 3 } Distancia media & Cambio de categoría & Definição circular/circularidade \\
\hline \multirow{5}{*}{ Distancia mínima } & $\begin{array}{l}\text { Cambio de característica definitoria } \\
\text { definitoria }\end{array}$ & $\begin{array}{l}\text { Dicionário de língua/dicionário } \\
\text { semasiológico }\end{array}$ \\
\cline { 2 - 3 } & Reducción & $\begin{array}{l}\text { Dicionário infantil/ dicionário escolar } \\
\text { infantil }\end{array}$ \\
\hline & Sinonimia & Macroestrutura/macro \\
\cline { 2 - 3 } & Variación formal & $\begin{array}{l}\text { Lexicografia pedagógica/ Lexicografia } \\
\text { didática }\end{array}$ \\
\hline
\end{tabular}

Finalmente, en el rango de distancia máxima se encontrarían las variantes donde se produce un cambio en la categoría conceptual representada en el núcleo. Consideramos que el cambio es mayor puesto que se produce una recategorización $y$, por tanto, se ubica al concepto en una posición distinta dentro de la estructura conceptual (variación intercategorial). Dentro de este grupo se incluirían los cambios de categoría (definição circular/circularidade), la hiperonimia e hiponimia-casos en que la variante representa un concepto genérico o específico con respecto al término de referencia, como rótulo/marca de uso -y cambio de configuración conceptual, donde se produce un cambio tanto en el núcleo como en el modificador -concepto genérico y característica(s) distintivas-, como verbete/artigo lexicográfico.

Puesto que esta clasificación opera mediante parejas denominativas, se comparó la información del término de referencia con cada una de las variantes denominativas introducidas por un marcador discursivo. Así en una serie enumerativa formada por un término de referencia y tres variantes denominativas, se comparó la información entre el término de referencia y cada una de las variantes (vd1+vd2+vd3) obteniendo así un total de 3 parejas denominativas. 


\section{Resultados}

En este apartado describimos los resultados del análisis semántico-cognitivo de la variación. En primer lugar describimos los patrones conceptuales de variación y en segundo lugar la distancia semántica entre variantes.

\subsection{Patrones conceptuales de variación denominativa}

Las 100 variantes denominativas -agrupadas en 49 grupos denominativos- pertenecen a 28 conceptos de la Lexicografía que, a su vez, se agrupan en 4 clases conceptuales: ramas de la Lexicografía (3 conceptos), obras lexicográficas (7 conceptos), componentes del diccionario ( 25 conceptos) y personas relacionadas con la actividad lexicográfica (2 conceptos).

A continuación, describimos las relaciones intratérmino y los patrones de especificación conceptual para cada clase, a fin de ver cuáles son las distintas perspectivas sobre los conceptos que se reflejan a través de este recurso denominativo. En la tabla 4 se muestran los patrones denominativos de los 3 conceptos que representan las ramas de la Lexicografía:

TABLA 4

Relaciones intratérmino y patrones de especificación conceptual de las ramas de la Lexicografía

\begin{tabular}{|l|l|l|l|l|}
\hline Punto de vista & $\begin{array}{l}\text { Patrón de } \\
\text { especificación }\end{array}$ & $\begin{array}{l}\text { Relación(es) } \\
\text { intratérmino }\end{array}$ & Frec. & Ejemplos \\
\hline PDV funcional & $\begin{array}{l}\text { Esp. del objeto } \\
\text { afectado }\end{array}$ & Objeto afectado & 4 & $\begin{array}{l}\text { Terminografia, lexicografia } \\
\text { terminológica }\end{array}$ \\
\cline { 2 - 5 } & Esp. de la función & Función & 3 & $\begin{array}{l}\text { Lexicografia de aprendizaje } \\
\text { Lexicografia pedagógica }\end{array}$ \\
\cline { 2 - 5 } & Esp. del destino & Destino & 4 & $\begin{array}{l}\text { Lexicografia projetada para } \\
\text { a escola, lexicografia infantil }\end{array}$ \\
\hline $\begin{array}{l}\text { PDV relacional/ } \\
\text { externo }\end{array}$ & Esp. de alcance & Alcance & 2 & $\begin{array}{l}\text { Lexicografia teórica, } \\
\text { lexicografia especializada }\end{array}$ \\
\hline
\end{tabular}

Como se muestra en la tabla, las 13 variantes que denominan las ramas de la Lexicografía seleccionan dos puntos de vista principales: el punto de vista funcional y el punto de vista relacional/externo. El punto de vista funcional, que destaca los aspectos funcionales de los conceptos se despliega en la especificación conceptual del objeto afectado, que distingue el concepto a partir del objeto sobre el que recae la actividad (lexicografia terminológica); en la especificación de la función, donde se selecciona como característica distintiva la actividad funcional a la que está destinado el concepto del núcleo (lexicografia pedagógica); o en la especificación del destino, es decir, en qué o quiénes son los destinatarios finales del trabajo lexicográfico (por ejemplo la escuela en Lexicografía projetada para a escola y los niños en Lexicografía infantil). Por otro lado, el punto de vista relacional externo, que destaca las características externas o contextuales de los conceptos (tiempo, alcance, agente, lugar, etc.), está presente en dos variantes de nuestro corpus, que especifican el alcance o cobertura de la actividad lexicográfica: los aspectos teóricos (Lexicografía teórica) o las áreas especializadas (Lexicografía especializada). 
Como se observa en nuestro análisis, a través de la variación denominativa se muestran diferentes perspectivas de conceptualización de la actividad lexicográfica, que se emplean para su clasificación en ramas. En algunos casos, las variantes de un mismo concepto seleccionan distintas perspectivas de clasificación (por ej. Lexicografía especializada/Lexicografía terminológica), en otros seleccionan matices distintos dentro de la misma perspectiva (lexicografia pedagógica/lexicografia de aprendizagem) y en otros casos de variación formal no hay diferencias en el criterio de clasificación seleccionado (terminografia/lexicografia terminológica).

A continuación, pasamos a describir los patrones de especificación conceptual de las 36 variantes que representan los 7 conceptos de obras lexicográficas de nuestro corpus (Tabla 5).

TABLA 5

Relaciones intratérmino y patrones de especificación conceptual de las obras lexicográficas

\begin{tabular}{|c|c|c|c|c|}
\hline Punto de vista & $\begin{array}{l}\text { Patrón de } \\
\text { especificación }\end{array}$ & $\begin{array}{l}\text { Relación(es) } \\
\text { intratérmino }\end{array}$ & Frec. & Ejemplos \\
\hline \multirow[t]{4}{*}{ PDV funcional } & \multirow[t]{2}{*}{ Esp. de la función } & Función & 5 & Dicionário de usos \\
\hline & & $\begin{array}{l}\text { Función + cont. inf. } \\
\text { y repr. + origen }\end{array}$ & 2 & $\begin{array}{l}\text { Dicionário pedagógico da } \\
\text { língua materna }\end{array}$ \\
\hline & \multirow[t]{2}{*}{ Esp. del destino } & Destino & 6 & Dicionário infantil \\
\hline & & Destino+tamaño & 1 & Dicionário escolar mini \\
\hline \multirow[t]{4}{*}{ PDV parte/todo } & $\begin{array}{l}\text { Esp. de los } \\
\text { constituyentes }\end{array}$ & Constituyentes & 3 & Léxico, vocabulário \\
\hline & \multirow{3}{*}{$\begin{array}{l}\text { Esp. del contenido } \\
\text { informativo y } \\
\text { representación }\end{array}$} & Cont. inf. y rep. & 3 & Dicionário terminológico \\
\hline & & $\begin{array}{l}\text { Cantidad+cont. inf. } \\
\text { y repr. +alcance }\end{array}$ & 1 & $\begin{array}{l}\text { Dicionário unilíngue da } \\
\text { língua geral }\end{array}$ \\
\hline & & $\begin{array}{l}\text { Cont. inf. y rep.+ } \\
\text { alcance }\end{array}$ & 2 & Dicionário de língua geral \\
\hline \multirow{3}{*}{$\begin{array}{l}\text { PDV de los } \\
\text { atributos } \\
\text { internos }\end{array}$} & $\begin{array}{l}\text { Esp. de los } \\
\text { atributos formales }\end{array}$ & Atributos formales & 1 & Dicionário semasiológico \\
\hline & Esp. del tamaño & Tamaño & 2 & Minidicionário \\
\hline & Esp. de la cantidad & Cantidad & 6 & Dicionário bilíngue \\
\hline \multirow{3}{*}{$\begin{array}{l}\text { PDV relacional/ } \\
\text { externo }\end{array}$} & Esp. de estatus & Estatus & 1 & Dicionário padrão \\
\hline & Esp. del tiempo & Tiempo & 1 & Pré-dicionário \\
\hline & Esp. del alcance & Alcance & 2 & Dicionário geral \\
\hline
\end{tabular}

En las variantes que pertenecen a la clase conceptual de la obras lexicográficas, se manifiestan cuatro puntos de vista: funcional, parte/todo, atributos internos y relacional/externo.

Desde el punto de vista funcional se resaltan 2 tipos de patrones de especificación: (a) función, como en el ejemplo dicionário de usos (obra cuya función es orientar en cuanto al uso) o en el ejemplo dicionário pedagógico da língua materna, donde también aparece mencionado el contenido informativo representado (lengua) y el origen de este contenido (materna); (b) destino, entendido como el lugar o persona a quien se destina la obra (dicionário infantil), el cual viene en un caso complementado por el tamaño (dicionário escolar mini).

Desde el punto de vista parte/todo, los patrones conceptuales especifican los constituyentes, como por ejemplo en léxico o vocabulário, esto es, conjunto de las 
unidades léxicas o vocablos de una lengua; y también encontramos variantes donde se especifica el contenido informativo y representación (dicionário de língua), que puede a su vez complementarse con otras relaciones intratérmino como alcance (dicionário de língua geral) o cantidad, como en dicionário unilíngue de língua geral, donde se especifica el número de lenguas contenidas en el diccionario.

Desde el punto de vista de los atributos internos, las variantes seleccionan cuatro patrones de especificación conceptual: (a) especificación de los atributos formales, como en el caso de dicionário semasiológico que se define a partir de la organización formal; (b) tamaño (minidicionário) y (c) cantidad, que alude a la cantidad de lenguas contenidas en el diccionario (dicionário bilíngue).

Finalmente, desde el punto de vista relacional/externo, encontramos variantes donde se especifica el estatus de la obra lexicográfica, como en dicionário padrão, donde se define a partir de la posición de modelo que detenta la lengua representada; el tiempo o etapa donde se sitúa (pré-dicionário) y el alcance (dicionário geral).

A continuación pasamos a describir los patrones conceptuales de variación de las 47 variantes que representan los 16 conceptos de componentes del diccionario en el corpus analizado (Tabla 6).

TABLA 6

Relaciones intratérmino y patrones de especificación conceptual de los componentes del diccionario

\begin{tabular}{|c|c|c|c|c|}
\hline Punto de vista & Patrón de especificación & $\begin{array}{l}\text { Relación(es) } \\
\text { intratérmino }\end{array}$ & Frec. & Ejemplos \\
\hline PDV igualdad & Esp. de yuxtaposición & Yuxtaposición & 1 & Palavra-entrada \\
\hline \multirow[t]{4}{*}{ PDV funcional } & Esp. del rol & Rol & 1 & Enunciado definitório \\
\hline & Esp. de manera & Manera & 10 & $\begin{array}{l}\text { Definição sinonímica, } \\
\text { Definiçãa analítica }\end{array}$ \\
\hline & Esp. de la función & Función & 1 & Marca de uso \\
\hline & Esp. del uso & Uso & 4 & Remissão \\
\hline \multirow[t]{2}{*}{ PDV parte/todo } & Esp. del todo & Partitiva & 6 & $\begin{array}{l}\text { Item lexical, } \\
\text { cabeça do verbete }\end{array}$ \\
\hline & Esp. de los constituyentes & Constituyentes & 2 & Nomenclatura \\
\hline \multirow{4}{*}{$\begin{array}{l}\text { PDV de los } \\
\text { atributos } \\
\text { internos }\end{array}$} & Esp. del tamaño & Tamaño & 4 & Macroestrutura \\
\hline & Esp. de la naturaleza & Naturaleza & 4 & Termo \\
\hline & $\begin{array}{l}\text { Esp. del concepto } \\
\text { atribuido }\end{array}$ & Concepto atribuido & 3 & Estrutura do verbete \\
\hline & Esp. de atrib. Formales & Atributos formales & 2 & Rótulo \\
\hline \multirow{4}{*}{$\begin{array}{l}\text { PDV relacional } \\
\text { externo }\end{array}$} & \multirow[t]{2}{*}{ Esp. de estatus } & Estatus & 6 & forma canônica \\
\hline & & Estatus + lugar & 1 & $\begin{array}{l}\text { Entrada canônica nos } \\
\text { dicionários }\end{array}$ \\
\hline & Esp. del lugar & Lugar & 1 & Entrada \\
\hline & Esp. del alcance & Alcance & 1 & Dominio \\
\hline
\end{tabular}

Las variantes pertenecientes a esta clase conceptual presentan una mayor diversidad de puntos de vista y relaciones intratérmino, ya que también es una clase con mayor variabilidad interna. Desde el punto de vista de la igualdad encontramos la variante palavra-entrada, que expresa una relación de yuxtaposición entre dos constituyentes que tienen igual estatus. Desde el punto de vista funcional, el más frecuente 
para esta clase, se observan cuatro tipos de especificaciones conceptuales: el rol o papel que representa el modificador con respecto al núcleo (enunciado definitório), la manera, que está presente en los tipos de definiciones e indica la manera cómo se realizan estos enunciados (definición sinonímica, definición analítica); la función (marca de uso), que indica la actividad funcional para la que está destinada el concepto; y el uso (remissão), que refleja la acción realizada por el concepto (remitir). Desde el punto de vista parte/todo encontramos variantes que reflejan una relación partitiva, donde el modificador representa la totalidad a la que pertenece el núcleo (item lexical); o donde se especifican los constituyentes (por ej. nomenclatura, que indica una lista compuesta de nombres). Del punto de vista de los atributos internos, algunas variantes especifican el tamaño (macroestructura), la naturaleza (termo), el concepto al que se atribuye la propiedad (estrutura do verbete) u otro atributo formal (rótulo). Con respecto a las variantes que especifican atributos externos o relacionales, encontramos la especificación del estatus (subentrada) -esta última complementada con lugar en entrada canônica nos dicionários- del lugar (entrada) o del alcance (dominio).

Finalmente, examinamos los patrones conceptuales de las 4 variantes pertenecientes a los dos conceptos de la clase conceptual de personas. En todos los casos se adopta un punto de vista funcional, pero en dos variantes del mismo concepto (leitor/ consulente) se especifica la función de las personas (leer o consultar la obra lexicográfica), y en el otro concepto ambas variantes especifican el producto de la actividad (persona que elabora diccionarios o léxicos).

TABLA 7

Relación intratérmino y patrones de especificación conceptual de las variantes: Personas

\begin{tabular}{|l|l|l|l|l|}
\hline Punto de vista & Patrón de especificación & $\begin{array}{l}\text { Relación(es) } \\
\text { intratérmino }\end{array}$ & Frec. & Ejemplos \\
\hline \multirow{2}{*}{ PDV funcional } & Esp. de la función & Función & 2 & Leitor/ Consulente \\
\cline { 2 - 6 } & Esp. del produto & Producto & 1 & Dicionarista/lexicógrafo \\
\hline
\end{tabular}

A modo de síntesis, en el análisis pudimos observar que hay ciertas preferencias por determinados patrones de especificación conceptual en la denominación de los conceptos de Lexicografía analizados. En primer lugar, el punto de vista funcional es el más productivo, pues aparece reflejado en $43 \%$ de las variantes analizadas (44 unidades). Dentro de este, la especificación de la función aparece con más frecuencia (13 unidades) en las denominaciones seguidas de la especificación de destino y manera, con 10 variantes cada una. En segundo lugar, encontramos reflejado el punto de vista de los atributos internos, con un $22 \%$ de los casos, (22 variantes) donde se especifica el tamaño (6 variantes), cantidad (6 variantes), el concepto atribuido u otros atributos formales (3 variantes). En tercer lugar, el punto de vista relacional/externo aparece seleccionado en 17 variantes (18\%) y distribuido en una variedad de patrones de especificación como alcance (8 variantes), estatus (6 unidades), lugar y tiempo, con 1 variante. Finalmente, de las variantes que reflejan el punto de vista parte/todo, destaca el patrón de especificación del contenido informativo y representación, especialmente productivo en la clase conceptual de obras lexicográficas, y el de la especificación del todo para la clase de componentes del diccionario.

La descripción de estos patrones nos permite, por un lado, identificar qué informaciones conceptuales seleccionan preferentemente las denominaciones de 
los conceptos pertenecientes a determinadas clases conceptuales en el área de la Lexicografía. Pero, por otro lado, también nos permite apreciar las distintas perspectivas sobre un mismo concepto que se visualizan a través de la variación denominativa. Como se observa en las tablas, algunas variantes seleccionan un mismo patrón de especificación y no varían en la información conceptual reflejada, de modo que la presencia de variación no aporta información adicional (por ej. item lexical/item léxico); en otros casos, se selecciona un mismo patrón principal pero la variante añade información adicional (como en el caso de dicionário unilíngue y dicionário unilíngue da língua geral, donde a la cantidad se añade el contenido informativo y el alcance); en otros, las variantes alternan patrones conceptuales distintos, y por tanto reflejan criterios de clasificación alternativos (dicionário infantil/dicionário de aprendizagem). Las consecuencias cognitivas de estos tipos de variación serán distintas, puesto que la distancia semántica y el tipo de información proporcionada por este recurso varía. A través de la clasificación de las variantes de acuerdo a la distancia semántica, que proponemos en el siguiente apartado, podremos cuantificar la presencia de estos diferentes tipos de variantes y obtendremos una medida de la función cognitiva de la variación denominativa.

\subsection{Distancia semántica entre las variantes}

La tabla 8 muestra la clasificación de las $83^{6}$ parejas denominativas de acuerdo a la distancia semántica:

TABLA 8

Clasificación semántica de la variación denominativa

\begin{tabular}{|c|c|c|c|}
\hline Clasificación & Frec. (\%) & Ejemplo & \multirow{4}{*}{$\begin{array}{l}\text { Distancia } \\
\text { máxima } \\
42 \%\end{array}$} \\
\hline $\begin{array}{l}\text { Cambio de configura- } \\
\text { ción conceptual }\end{array}$ & $27(32 \%)$ & $\begin{array}{l}\text { Entrada/lema } \\
\text { Verbete/artigo lexicográfico }\end{array}$ & \\
\hline Hiperónimo/hipónimo & $3(4 \%)$ & $\begin{array}{l}\text { Marcas de uso/rubrica } \\
\text { Rótulos/ marcas de uso }\end{array}$ & \\
\hline Cambio de categoría & $5(6 \%)$ & $\begin{array}{l}\text { Definição circular/circularidade } \\
\text { Entrada de verbete/ palavra-entrada }\end{array}$ & \\
\hline $\begin{array}{l}\text { Cambio de característica } \\
\text { distintiva }\end{array}$ & $28(34 \%)$ & $\begin{array}{l}\text { Dicionário de língua/dicionário semasiológico } \\
\text { Dicionário infantil/dicionário escolar }\end{array}$ & \multirow{3}{*}{$\begin{array}{l}\text { Distancia } \\
\text { media } \\
43 \%\end{array}$} \\
\hline $\begin{array}{l}\text { Cambio de característica } \\
\text { no distintiva }\end{array}$ & $4(5 \%)$ & $\begin{array}{l}\text { Dicionário infantil/ dicionário escolar infantil } \\
\text { Dicionário escolar mini/ minidicionário }\end{array}$ & \\
\hline Reducción & $3(4 \%)$ & $\begin{array}{l}\text { Entrada de verbete/entrada } \\
\text { Macroestrutura/macro }\end{array}$ & \\
\hline Sinonimia & $6(7 \%)$ & $\begin{array}{l}\text { Nomenclatura/nominata } \\
\text { Lexicografia pedagógica/ Lexicografia didática }\end{array}$ & \multirow{2}{*}{$\begin{array}{l}\text { Distancia } \\
\text { mínima } \\
15 \%\end{array}$} \\
\hline Variación formal & $7(8 \%)$ & $\begin{array}{l}\text { Dicionários pedagógicos en língua materna/ } \\
\text { DPLMs } \\
\text { Item lexical/item léxico }\end{array}$ & \\
\hline
\end{tabular}

Como se observa en la tabla anterior, la variación denominativa explícita en el corpus analizado se concentra especialmente en los rangos de máxima y media distancia semántica, con un $42 \%$ y $43 \%$ de ocurrencias respectivamente. Los tipos más frecuentes son, en primer lugar, la variación por cambio de característica distintiva (34\%) y el cambio de configuración conceptual (32\%). En estos casos, la variación 
denominativa explícita cumple una función informativa, pues refleja alternativas de categorización simultáneas y proporciona diferentes dimensiones del contenido de los conceptos especializados. En cambio, el número de variantes con mínima distancia semántica, como los sinónimos (6 oc.) y las variantes formales (7 oc.) solo concentran el 15\% de las 83 parejas denominativas analizadas. Consideramos que, al tratarse de un cambio formal sin cambio semántico, esta variación no tiene una función cognitiva.

A continuación, describimos el tipo de información aportada por cada categoría de la clasificación con ejemplos del corpus.

Cambio de configuración conceptual: Como señalamos anteriormente, se considera cambio de configuración conceptual los casos en que las variantes seleccionan tanto en la base como en la extensión conceptos no relacionados, lo cual produce una recategorización y la selección de características distintivas distintas.

$3)^{(\mathrm{TR})}$ Entrada [o mesmo que, ${ }^{(\mathrm{VD} 1)}$ lema, ${ }^{(\mathrm{VD} 2)}$ cabeça do verbete, [...]]. É a palavra ou expressão que encabeça o verbete, sendo o elemento a ser definido ou explicado.

(Rangel y Bagno 2006: 151)

Como se ve en el ejemplo, el término de referencia entrada nos informa sobre el lugar que ocupa este elemento en la microestructura del artículo lexicográfico. Lema, por su parte, identifica el elemento a partir de su naturaleza, es decir, una unidad léxica en su forma canónica no flexionada. Finalmente, la variante cabeça do verbete indica en el núcleo la posición, en este caso en la parte superior (cabeça), y, en el modificador, el elemento del que forma parte, esto es, el artículo lexicográfico (verbete). Consideramos que el cambio de configuración conceptual posee el grado de informatividad más alto de nuestra clasificación, ya que presenta información intercategorial e intracategorial y proporciona visiones distintas pero complementarias de un mismo concepto especializado. Es el segundo tipo más frecuente en el corpus, con 54 variantes y un $32 \%$ de los casos analizados.

Hiperónimo/hipónimo: En la siguiente categoría, la variante representa un concepto hiperónimo o hipónimo con respecto al término de referencia. Como se aprecia en el ejemplo, la variante rubrica es un concepto más genérico que marcas de uso, que especifica en la extensión la función de este elemento identificativo, a saber, orientar sobre el uso de una determinada unidad léxica en el diccionario.

4) As ${ }^{(\mathrm{TR})}$ marcas de uso também chamadas de ${ }^{(\mathrm{VDl})}$ rubricas, tem a função de caracterizar palavras e expressões, indicando, condicionando e, por vezes, restringindo seu uso e emprego no contexto discursivo.

(Krieger 2013: 80) $)^{8}$

Consideramos que la variación hiperonímica o hiponímica aporta información intercategorial, concretamente sobre las relaciones taxonómicas entre conceptos dentro de la estructura conceptual. Puesto que la variación se da en la base del término, se produce una recategorización del concepto, por lo tanto este tipo de variante se situaría en la categoría de distancia máxima. Este tipo de variación, que es común como recurso de correferencia léxica anafórica y catafórica (Halliday y Hasan 1976), es residual en nuestro corpus de trabajo (6 ocurrencias, $4 \%$ ); creemos que se debe a la presencia del marcador explícito, que no anticipa prototípicamente una relación de inclusión sino de equivalencia. 
Cambio de categoría: En este tipo de variación, la variante selecciona una información conceptual que ya estaba expresada en el término de referencia, pero modifica el estatus de la misma al pasar de constituir el concepto genérico a una característica distintiva.

5) ${ }^{(\mathrm{TR})}$ Definição circular [também denominada ${ }^{(\mathrm{VDl})}$ circularidade]: ocorre quando uma palavra-entrada é definida por um sinônimo, e vice-versa. O sinônimo definidor, quando consultado na entrada correspondente, tem seu sentido definido por aquela palavra que ele próprio definiu.

(Rangel y Bagno 2006: 146) ${ }^{9}$

Como se muestra en el ejemplo, la variante de referencia definição circular menciona en la base el tipo de enunciado (definición) y, en la extensión, la característica distintiva, a saber, la manera de la definición por autorreferencia, ya que ese tipo de definición remite a sí misma por el uso de sinónimos. En la variante circularidade está presente la misma característica distintiva, pero recategorizada como propiedad que presentan este tipo de definiciones. En este caso la mención a la definición desaparece, pero queda implícita en el concepto de circularidad (ya que en el contexto solo se aplica a los tipos de definiciones). Este tipo de variante tampoco es frecuente en nuestro corpus, con 10 ocurrencias (8\%).

Cambio de característica distintiva: En este tipo de variación, al igual que todos los que se sitúan en la distancia media dentro de nuestra clasificación, se mantiene el concepto representado en la base del término y solo se altera la característica distintiva, representada en la extensión. Entendemos por distintiva la característica que permite diferenciar el concepto de sus cohipónimos dentro de la estructura conceptual. Tal y como se muestra en siguiente ejemplo, las variantes dicionário geral de língua y dicionário semasiológico comparten la misma base que el término de referencia dicionário padrão, y por lo tanto son categorizadas como "tipos de obras que recogen el vocabulario de una lengua acompañadas de informaciones sobre su significado y uso".

6) Em todas as taxonomias já mencionadas, há consenso sobre a existência de um genótipo chamado por Biderman (1998, p. 129) “(TR) dicionário padrão” ou “(VD1) dicionário geral de língua". Respeitosamente, atrevemo-nos a chamar esse tipo de dicionário de "(VD2) dicionário semasiológico", já que a sua tarefa básica é a de fornecer significados.

(Bugueño-Miranda y Farias 2011: 46) ${ }^{10}$

Sin embargo, varía el patrón de especificación conceptual, que se emplea para diferenciar ese diccionario de otro tipo de diccionarios: padrão indica el estatus prescriptivo del diccionario, que tiene por función orientar sobre el uso de la norma estándar; en la VD1 el diccionario se define por el contenido informacional y el alcance (de la lengua general) y en la VD2 se define a partir de una característica formal, la organización u orientación semasiológica, es decir partiendo del significante para el significado. Este tipo de variación es el más frecuente en nuestro corpus, con 56 ocurrencias (34\%). Se asocia a la multidimensionalidad de las estructuras conceptuales (Bowker 1998; Tercedor 2011), puesto que un concepto admite diferentes criterios de clasificación dentro de un mismo ámbito. Como se observa en el ejemplo, no se trata de características opuestas, sino de distintos criterios de clasificación que conviven de manera complementaria en el sistema conceptual. 
Cambio de característica no distintiva: En este caso, la variante denominativa añade o suprime una característica del concepto con respecto al término de referencia, pero en este caso, se trata de una característica que no se considera definitoria sino que complementa la información del concepto, tal y como se muestra en el siguiente ejemplo.

7) Como se observa, a terminologia não é fixa nas línguas estrangeiras e em português oscila entre ${ }^{(\mathrm{VDR} 1)}$ Dicionário Escolar, ${ }^{(\mathrm{VDR} 2)}$ Infantil, ${ }^{(\mathrm{VDR} 3)}$ Escolar Infantil, ${ }^{(\mathrm{VDR} 4)}$ de Iniciação, ${ }^{(V D R 5)}$ Pré- dicionários. Como já apontado anteriormente, darei preferência à utilização do termo ${ }^{(\mathrm{TR})}$ Dicionário Infantil.

(Zavaglia 2010: 40-41) $)^{11}$.

En el ejemplo, el término de referencia refleja únicamente el patrón de especificación conceptual principal, a saber, los destinatarios del diccionario (infantil); en la variante denominativa se complementa la caracterización etaria de los destinatarios añadiendo el lugar al que está destinado (escolar). En el corpus la presencia de este tipo de variación es modesta (8 ocurrencias, $5 \%$ ), creemos que, por el carácter redundante del par denominativo, donde se repiten los patrones de especificación conceptual entre término de referencia y variante.

Reducción: En el caso de variación por reducción, se suprime la extensión del sintagma terminológico. En términos conceptuales, la variante resultante es un hiperónimo, pero la consecuencia cognitiva es menor que en el caso descrito más arriba porque mantiene la misma conceptualización que el término de referencia. Como se aprecia en el ejemplo, el autor incluye la variante entrada precedida del adverbio simplesmente, sugiriendo que la información sobre el concepto del que forma parte (verbete) expresada en el término de referencia se puede deducir por el contexto:

8) É sinônimo de ${ }^{(\mathrm{TR})}$ entrada de verbete, (VDRI) palavra-entrada ou simplesmente (VDR2) entrada. O termo não é muito comum no Brasil, mas já foi utilizado por Biderman (1984: 6), e depois, entre outros, além de Biderman (2000), por Carvalho (2001).

(Welker 2004: 33) ${ }^{12}$

La reducción es uno de los tipos de variación intratextual mejor descritos en la bibliografía como recurso para evitar la repetición (Collet 2003). Sin embargo, en el caso de la variación denominativa explícita como la que se analiza en este artículo, se incurre deliberadamente a la repetición del concepto a través de alternativas denominativas, lo cual podría explicar la baja incidencia de este tipo de variación en nuestro corpus (4\%).

Sinonimia: En este caso, las variantes denominativas constituyen sinónimos léxicos de los términos de referencia. La información sobre el concepto no varía, por lo que su función cognitiva sería mínima. Como se aprecia en el ejemplo, la información conceptual que refleja el término de referencia lexicografia pedagógica y la variante lexicografia didática es idéntica: en ambos casos se conceptualiza esta rama de la Lexicografía a partir de su función, la enseñanza.

9) De fato, há um crescimento editorial no plano da ${ }^{(\mathrm{TR})}$ lexicografia pedagógica ou (VDR1) lexicografia didática, que se destina aos estudantes no aprendizado de sua língua materna.

(Santiago 2012: 3) $)^{13}$

La sinonimia constituye el 7\% de las variantes analizadas en nuestro corpus. En la mayoría de los casos se trata de variantes poliléxicas, donde alguno de los elementos 
denominativos es sinónimo y los otros se repiten, como en el ejemplo presentado. Sin embargo, tambien hay casos de unidades monoléxicas sinónimas, como por ejemplo nomenclatura/nominata.

Variación formal: En este caso se produce un cambio formal inferior al nivel léxico, por ejemplo, ortográfico, morfológico o morfosintáctico. En este caso se considera que la distancia semántica es mínima ya que el contenido léxico no se modifica, y por lo tanto la información conceptual se mantiene intacta. Como se muestra en el ejemplo, la sigla DPLMs constituye una variante formal de dicionários pedagógicos en língua materna sin ninguna consecuencia cognitiva, ya que retoma mediante una forma abreviada la misma información conceptual que presentaba el término de referencia. En el corpus, nos encontramos 14 casos de variación formal (8\%), entre los que se encuentran siglas (dicionário infantil/DIS) y otras variantes morfológicas (item lexical/item léxico).

10) Por su vez, Welker (2008, p. 295) diz que “fazem parte dos ${ }^{(\mathrm{TR})}$ dicionários pedagógicos de língua materna ${ }^{(\mathrm{VD1})}(D P L M s)$ não somente os dicionário escolares como também, por um lado, os dicionários infantis (DIs)".

(Zavaglia 2010: 40) $)^{14}$

En síntesis, este análisis de la variación denominativa de acuerdo a la distancia semántica nos permite obtener una visión complementaria sobre la función de la variación denominativa explícita en los textos. Como vemos, la mayoría de variantes se concentran en la media y máxima distancia, de modo que, desde nuestra perspectiva, contribuyen a construir el conocimiento especializado en los textos y reflejan el carácter multidimensional de los conceptos, muchos de los cuales aceptan distintas perspectivas de categorización que no son excluyentes, sino complementarias.

\section{Conclusiones}

El objetivo de este trabajo fue analizar la función cognitiva de la variación denominativa explícita en el área de la Lexicografía, y más específicamente, su papel en la construcción y transmisión del conocimiento especializado en los textos. Para ello, llevamos a cabo un análisis semántico-cognitivo de las variantes denominativas relacionadas explícitamente mediante marcadores discursivos de equivalencia en un corpus de 300 textos académicos de Lexicografía brasileña. Este análisis nos permitió explorar dos aspectos: por un lado, la información conceptual expresada por medio de las variantes, y por otro, el tipo de cambio conceptual y la distancia semántica entre el término de referencia (primera ocurrencia del concepto) y las variantes denominativas.

En relación con el primer aspecto, identificamos los patrones de especificación conceptual de las variantes pertenecientes a una misma clase conceptual, es decir, las dimensiones del contenido conceptual reflejadas en las variantes. Este análisis arrojó resultados interesantes sobre el comportamiento denominativo de los conceptos de la Lexicografía. En primer lugar, vimos que existen preferencias hacia la selección de determinados aspectos del contenido conceptual según la clase: en las cuatro clases analizadas el punto de vista funcional fue el predominante, que se manifiesta en la especificación de la función, destino, uso, manera u objeto afectado principalmente. Sin embargo, en otras clases como las obras lexicográficas o los componentes del diccionario, también se seleccionan atributos internos o externos o se especifica la 
relación entre el todo y sus partes. En algunos casos, las variantes de un mismo concepto seleccionan diferentes aspectos del contenido conceptual y reflejan diferentes criterios de clasificación posibles para un mismo concepto, lo cual a nuestro juicio refleja la naturaleza multidimensional de los conceptos y las estructuras conceptuales. Este fenómeno proporciona una representación enriquecida de los conceptos y, por ello, estimamos que la variación desempeña una función cognitiva pues contribuye a la construcción y transmisión del conocimiento especializado en los textos.

En la segunda parte del análisis examinamos específicamente qué tipo de cambio conceptual se producía entre el término de referencia y la(s) variante(s) denominativa(s) y lo organizamos de acuerdo a una escala de distancia semántica, con el objetivo de cuantificar la función cognitiva de la variación. Este análisis nos permitió determinar que la mayor parte de los casos de variación en nuestro corpus implican un cambio en la manera en que se proyecta el concepto ( $85 \%$ de las variantes se encuentran en el rango de distancia media y máxima). Por otro lado, al analizar específicamente la función de cada uno de los 8 tipos de variación presentes en el corpus, pudimos observar que la variación proporciona información intracategorial e intercategorial sobre el concepto, es decir, información sobre las características internas del concepto y sobre su relación con otros conceptos del ámbito respectivamente.

Creemos que este análisis semántico-cognitivo confirma dos tesis fundamentales presentadas en el marco teórico: por un lado, la variación es el reflejo lingüístico de la flexibilidad y multidimensionalid de los conceptos y estructuras conceptuales y de su carácter situado. Como hemos visto, a menudo conviven diferentes perspectivas de categorización de un mismo concepto, lo cual se asocia con factores cognitivos y contextuales. Por otro lado, la variación denominativa desempeña una función cognitiva en la construcción y transferencia del conocimiento especializado, ya que a través de ella los conceptos van desplegando su contenido y relaciones dentro de la estructura del ámbito. Con respecto a este último punto, somos conscientes de las limitaciones que tiene analizar la función cognitiva de la variación en corpus, ya que solamente se puede observar el contenido semántico reflejado en los textos. Sin embargo, si asumimos que las estructuras lingüísticas son un reflejo de las estructuras mentales, tesis fundamental de la lingüística cognitiva, consideramos que este estudio constituye un primer paso para explorar la función cognitiva de la variación. Sin duda, en un futuro este estudio tiene que complementarse con otras investigaciones en que se trabaje con un corpus de mayor tamaño, con más datos de frecuencias y también trabajos en que se analice el efecto de la variación en la comprensión del conocimiento especializado por parte de los receptores de los textos.

\section{NOTAS}

1. Este estudio se realizó en el marco del proyecto de investigación postdoctoral DI/2016 titulado "La variación denominativa y conceptual en la lexicografía brasileña y su representación en un diccionario especializado destinado a estudiantes universitarios," financiado por la Pontificia Universidad católica de Valparaíso en el periodo 2016-2017.

2. Consideramos este recorte temporal porque fue a partir de la década de 1980, concretamente con la publicación de los primeros textos de Biderman en 1984, que se impulsaron los estudios sobre Lexicografía en uso en Brasil.

3. El programa Wordsmith Tools (http://www.lexically.net/wordsmith/) es una herramienta de explotación de corpus y fue desarrollada por el lingüista británico Mike Scott en la Universidad de Liverpool con su primera versión 1.0 en el año 1996, mientras que Terminus (http://terminus.iula. 
upf.edu) es una estación de trabajo específicamente diseñada para el trabajo terminológico, fue creada y desarrollada por lo Grupo IULATERM del Institut Universitari de Lingüística Aplicada de la Universitat Pompeu Fabra.

4. Zavaglia, Claudia (2010): Dicionários Infantis: uma análise de suas microestruturas [Diccionarios Infantiles: un análisis de sus microestructuras]. San José del Río Negro: UNESP.

5. Welker, Herbert Andréas (2004): Dicionários: uma pequena introdução à lexicografia [Diccionarios: una pequeña introducción a la lexicografía]. Brasília: Thesaurus.

6. Si bien el número total de variantes denominativas es 100, para el análisis de la distancia semántica se analizaron las variantes en parejas (término de referencia + variante), sumando 83 parejas denominativas.

7. Rangel, Egon y Bagno, Marcos (2006): Dicionários em sala de aula [Diccionarios en el aula]. Brasília: Ministério da Educação, Secretaria de Educação Básica.

8. Krieger, Maria da Graça (2013): Dicionário em sala de aula: guia de estudos e exercícios [Diccionario en el aula: guía de estudios y ejercicios]. Rio de Janeiro: Lexicon.

9. Vease nota 7.

10. Bugueño-Miranda, Felix y Farias, Virginia Sita (2011): Da microestrutura em dicionários semasiológicos do português e seus problemas [Microestructura en los diccionarios semasiológicos de portugués y sus problemas]. Estudos da Lingua(gem). 9(1):39-69.

11. Vease nota 4.

12. Vease nota 5.

13. Santiago, Marcio (2012): Análises contrastivas de microestruturas em dicionários escolares [Análisis de las microestructuras en los diccionarios escolares]. Pesquisas em Discurso Pedagógico. 2012(1):14 p. Consultado el 12 de agosto de 2016, <https://www.maxwell.vrac.puc-rio.br/20000/ 20000.PDFXXvmi=>.

14. Vease nota 4.

\section{REFERENCIAS}

BARsalou, Lawrence (2003): Situated simulation in the human conceptual system. Language and Cognitive Processes. 18(5-6):513-562.

Bertaccini, Franco y Matteucci, Alessandra (2005): L'approche variationniste à la pratique terminologique d'entreprise. Meta. 50(4):CD-ROM.

Bowker, Lynne (1998): Variant terminology: frivolity or necessity? In: Thierry Fontenelle, Philippe Hiligsmann, Archibald Michiels, et al., eds. Actes EURALEX'98. (Huitième Congrès International de Lexicographie, Liège, 4-8 de agosto de 1998). Vol. II. Liège: Université de Liège, 487-495.

Bowker, Lynne y Hawkins, Shane (2006): Variation in the organisation of medical terms: Exploring some motivations for term choice. Terminology. 12(1):79-110.

CABRÉ, María Teresa (1999): La Terminología: representación y comunicación: elementos para una teoria de base comunicativa y otros artículos. Girona: Documenta Universitaria.

CABré, María Teresa (2008): El principio de poliedricidad: la articulación de lo discursivo, lo cognitivo y lo lingüístico em Terminología. Ibérica. 16:9-36.

Ciapuscio, Guiomar (2003): Textos especializados y terminología. Barcelona: IULA-UPF.

Collet, Tanja (2003): A two-level grammar of the reduction processes of french complex terms in discourse. Terminology. 9(1):1-27.

Contente, Madalena y Magalhães, João (2005): Sinonimologia e tipologia contrastiva da sinonímia terminológica em medicina [Sinonimología y tipología contrastiva de la sinonimia terminológica en medicina]. Revista Debate Terminológico. 1:18 p. Consultado el 3 de septiembre de 2016, <https://seer.ufrgs.br/riterm/article/view/21289/12269>.

Costa, Lucimara Alves (2015): Reflexões sobre a variação terminológica na lexicografia corrente no Brasil e a construção das bases teórico-metodológicas para o dicionário de Lexicografia brasileira [Reflexiones sobre la variación terminológica en la lexicografía corriente en Brasil y la construcción de las bases teórico-metodológicas para el diccionario de Lexicografía brasileña]. Tesis de doctorado no publicada. San José del Río Negro/Barcelona: Universidade Estadual Paulista/Universitat Pompeu Fabra. 
Diki-KidiRI, Marcel (2008): Le vocabulaire scientifique dans les langues africaines: pour une approche culturelle de la terminologie. Paris: Karthala.

FABER, Pamela, ed. (2012): A Cognitive Linguistics View of Terminology and Specialized Language. Berlin/Boston: Mouton de Gruyter.

Faber, Pamela, Márquez, Carlos y Vega, Miguel (2005): Framing terminology: A processpriented approach. Meta. 50 (4):CD-ROM.

Faber, Pamela, Martínez, Silvia, Prieto, Maria Rosa, et al. (2006): Process-oriented terminology management in the domain of Coastal Engineering. Terminology. 12(2):189-213.

FaUlSTiCH, Enilde (1998): Variação terminológica: algumas tendências no português do Brasil [Variación terminológica: algunas tendencias en portugués de Brasil]. In: Pamela FABER, ed. Lèxic, corpus i diccionaris. Cicle de conferències 96-97 [Léxico, corpus y diccionarios. Ciclo de conferencias 96-97]. Barcelona: Institut Universitari de Lingüística Aplicada/ Universitat Pompeu Fabra, 141-154.

Faulstich, Enilde (2000): Principles formels et fonctionnels de la variation en terminologie. Terminology. 5(1):93-106.

Fernández Polo, Francisco Javier (1999): Traducción y retórica contrastiva. A propósito de la traducción de textos de divulgación científica del inglés al español. Santiago de Compostela: Universidad de Santiago de Compostela.

FERNÁNDEZ-SILVA, Sabela (2011): Variación terminológica y cognición: factores cognitivos en la denominación del concepto especializado. Barcelona: IULA-TDX.

FernándeZ-Silva, Sabela (2016): The cognitive and rhetorical role of term variation and its contribution to knowledge construction in research articles. Terminology. 22(1):52-79.

Fernández-Silva, Sabela, Freixa, Judit y Cabré, Maria Teresa (2011): A proposed method for analysing the dynamics of cognition through term variation. Terminology. 17(1):49-74.

Fillmore, Charles (1985): Frames and the semantics of understanding. Quaderni di semantica. $6(2): 222-254$.

FreiXa, Judit (2002): La variació rerminològica: Anàlisi de la variació denominativa en textos de diferent grau d'especialització de l'àrea de medi ambient [La variación terminológica: Análisis de la variación denominativa en textos de diferente grado de especialización del área de medio ambiente]. Barcelona: IULA.

FreiXA, Judit (2005): Variación terminológica: ¿por qué y para qué? Meta. 50(4):CD-ROM.

FreiXa, Judit (2006): Causes of denominative variation in terminology: A typology proposal. Terminology. 12(1):51-77.

GAUdin, François (1993): Pour une socioterminologie: des problemes sémantiques aux pratiques institutionnelles. Rouen: Université de Rouen.

Halliday, Michael y Hasan, Ruqayia (1976): Cohesion in English. London: Longman.

Jesus, Ana Maria y Barros, Lídia (2005): A variação terminológica em português no domínio da Dermatologia [El cambio terminológico en portugués en el campo de la dermatología]. Signótica. 17(2):165-189.

Kageura, Kyo (2002): The Dynamics of Terminology: A Descriptive Theory of Term Formation and Terminological Growth. Amsterdam/Philadelphia: John Benjamins.

Kocourek, Rostislav (1991): La langue française de la technique et de la science: vers une linguistique de la langue savante. Weisbaden: Oscar Brandstetter.

Krieger, Maria da Graça y Santiago, Márcio (2014): Terminologia médica e variação [Terminología médica y variación.]. In: Dermeval da Hora, Juliene Lopes Ribeiro Pedrosa y Rubens Marques de Lucena, eds. Anais da ALFAL 2014 [Anales de la ALFAL 2014]. (XVII Congreso Internacional de la Asociación de Lingüística y Filología de América Latina, João Pessoa, 14-18 de julio de 2014). João Pessoa: Ideia Editora, 4500-4509.

Lakoff, George y Johnson, Mark (1980): Metaphors We Live by. Chicago: University of Chicago Press.

Pecman, Mojca (2012): Tentativeness in Term Formation: A Study of Neology as a Rhetorical Device in Scientific Papers. Terminology. 18(1):27-58. 
Pecman, Mojca (2014): Variation as a Cognitive Device: How Scientists Construct Knowledge through Term Formation. Terminology. 20(1):1-24.

Rosch, Eleanor (1978): Principles of categorization. In: Eleanor RoACH y Barbara B. LLOYd, eds. Cognition and Categorization. Hillsdale: Erlbaum, 27-48.

Rogers, Margaret (2004): Multidimensionality in concepts systems: A bilingual textual perspective. Terminology. 10(2):215-240.

SEgHezZI, Natalia (2011): Variación terminológica y canal de comunicación. Estudio contrastivo de textos especializados escritos y orales sobre lingüística. Barcelona: IULA-TDX.

SuÁrez, Mercedes (2004): Análisis contrastivo de la variación denominativa en textos especializados: del texto original al texto meta. Barcelona: Institut Universitari de Lingüística Aplicada/Universitat Pompeu Fabra.

Temmerman, Rita (2000): Toward New Ways of Terminology Description: The Sociocognitive Approch. Amsterdam/Filadelfia: John Benjamins.

Tercedor, Maribel (2011): The Cognitive Dynamics of Terminological Variation. Terminology. 17(2):181-197.

Tercedor, Maribel y López, Clara Inés (2012): Access to health in an intercultural setting: The role of corpora and images in grasping term variation. Linguistica Antverpiensia. 11:247268.

WÜsTER, Eugen (1979/1998): Introducción a la teoría general de la terminología y a la Lexicografía terminológica. (Traducido por Anne-Cécile Nokerman) Barcelona: IULA-UPF. 